\title{
Incidence of antidepressant use among community dwellers with and without Parkinson's disease - a nationwide cohort study
}

Eerik Hentilä ${ }^{1,2}$, Miia Tiihonen ${ }^{1,2}$, Heidi Taipale ${ }^{1,2,3,4}$, Sirpa Hartikainen ${ }^{1,2}$ and Anna-Maija Tolppanen ${ }^{1,2^{*}}$

\begin{abstract}
Background: Antidepressant use is more common in people with Parkinson's disease (PD), but it is unknown when this difference emerges.

Methods: We studied the incidence of antidepressant use in six-month periods from 10 years before to 15 years after PD diagnosis in the nationwide register-based Finnish Study on Parkinson's disease (FINPARK). This study included 20,456 community dwellers with clinically verified PD diagnosed during 1996-2015 and 140,291 matched comparison persons.

Results: Altogether $44.3 \%$ of people with PD initiated antidepressants, compared to $25.0 \%$ of people without PD. The difference was largest 6 months before PD diagnosis (incidence rate ratio 5.28, 95\% Cl 4.80-5.80; 9.02 and 1.68 initiations/100 person-years in people with and without PD, respectively). The difference emerged already 7 years before the diagnosis and remained above the comparison group for most of the study period.

Conclusions: Persons with PD may have symptoms that require antidepressant treatment years before and after diagnosis. The symptoms needing antidepressant treatment may be clinical signs of possible PD and they should be considered as a need to assess clinical status in person diagnosed with PD.
\end{abstract}

Keywords: Parkinson's disease, Antidepressant, Incidence, Epidemiology, Cohort studies

\section{Background}

The average age when Parkinson's disease (PD) is diagnosed is approximately $65-70$ years [1]. Therefore, due to population aging, the prevalence of age-related disease including Parkinson's disease is expected to rise. Although PD is characterised by the motor symptoms, nonmotor symptoms are also common [2]. These symptoms range from functional somatic symptoms such as

\footnotetext{
* Correspondence: anna-maija.tolppanen@uef.fi

${ }^{1}$ School of Pharmacy, University of Eastern Finland, P.O. Box 1627, 70211 Kuopio, Finland

${ }^{2}$ Kuopio Research Centre of Geriatric Care, University of Eastern Finland, P.O. Box 1627, 70211 Kuopio, Finland

Full list of author information is available at the end of the article
}

constipation, to sleep disorders and to psychological symptoms, anxiety and mood disorders, including major and minor depression as well as clinically relevant depressive symptoms and dysthymia [3-6]. They can occur years before PD diagnosis $[3,4]$ and precede the motor symptoms [6].

Studies on people diagnosed with PD have demonstrated higher prevalence of mood disorders in comparison to general population [2, 7-9]. Depression is more frequent in people with $\mathrm{PD}$, although there is substantial variation in the estimated prevalence in people with $\mathrm{PD}$ (range 2.7-90\%) [7, 10-12]. This variation likely stems from differences between study populations, diagnosis

(c) The Author(s). 2021 Open Access This article is licensed under a Creative Commons Attribution 4.0 International License, which permits use, sharing, adaptation, distribution and reproduction in any medium or format, as long as you give appropriate credit to the original author(s) and the source, provide a link to the Creative Commons licence, and indicate if changes were made. The images or other third party material in this article are included in the article's Creative Commons licence, unless indicated otherwise in a credit line to the material. If material is not included in the article's Creative Commons licence and your intended use is not permitted by statutory regulation or exceeds the permitted use, you will need to obtain permission directly from the copyright holder. To view a copy of this licence, visit http://creativecommons.org/licenses/by/4.0/. The Creative Commons Public Domain Dedication waiver (http://creativecommons.org/publicdomain/zero/1.0/) applies to the data made available in this article, unless otherwise stated in a credit line to the data. 
and definition of depression and statistical measures used in individual studies [7]. According to a systematic review [7], the prevalence was lower in population-based studies than studies conducted in outpatient, inpatient or nursing home settings. In addition for major depressive disorder according to DSM criteria, studies using structured or semistructured interview have reported higher prevalence than those without a structured interview [7]. Anxiety is also common among persons with $\mathrm{PD}$, and it can occur independently or concomitantly with depression $[8,9]$.

Prevalence and incidence of antidepressants after initiation of dopaminergic antiparkinson drugs [13, 14] or inpatient or outpatient hospital visit with PD diagnosis [15] has been investigated. These studies demonstrated higher prevalence of antidepressant use among antiparkinson drug users than nonusers [13]. Antiparkinson drug users were also more likely to initiate antidepressants in comparison to nonusers of antiparkinson drugs [14]. One study compared the incidence of antidepressant use in cohort with hospitalisation with PD diagnosis to a cohort with hospitalisation with osteoarthritis diagnosis and showed that the incidence was higher in the PD cohort [15]. Due to the design of these previous studies (any dopaminergic antiparkinson drug use or admission to secondary healthcare with PD as the main diagnosis as proxy for $\mathrm{PD}$, and the applied timeframe for assessing antidepressant incidence) it is unknown when the difference emerges in relation to PD diagnosis, and how long the difference remains.

To summarise, previous studies have shown higher prevalence of nonmotor symptoms, including possible reasons for antidepressant initiation, and higher incidence and prevalence of antidepressants in people with hospital admission for PD or PD drug users. However, it is unknown when the difference emerges in relation to PD diagnosis. To illustrate this, we investigated the incidence of antidepressant use in six-month time windows from 10 years before to 15 years after PD diagnosis in a nationwide cohort, and compared the incidence to a matched cohort without PD.

\section{Methods}

The nationwide register-based Finnish Study on Parkinson's disease register-based FINPARK study includes 22, 189 people who received clinically confirmed PD diagnosis during the years 1996-2015 and were communitydwelling at the time of diagnosis.

People with PD diagnosis were identified from the Special Reimbursement Register maintained by the Social Insurance Institution of Finland (SII). Originally, 29,942 people eligible for reimbursement of antiParkinson drugs were identified, but as these drugs can also be used for other reasons, we excluded those who did not have ICD-10 code for PD (G20) recorded in the Special Reimbursement Register $(n=1244)$, those who were $<35$ years old at the time of PD diagnosis $(N=53)$ and those who had diagnoses whose symptoms may be confused with PD $(n=6456)$ within 2 years of PD diagnosis, which lead to a cohort of 22,189 people. The exclusion diagnoses are listed in Supplementary Table 1. These people were excluded, as diagnosis of PD and its differential diagnostics is challenging, and false diagnoses are common in the early phase $[16,17]$. The proportion of excluded people (25.9\%) in our study is within the range of estimated proportion of false diagnoses $[16,17]$.

The application for special reimbursement includes anamnesis of the patient and description of the characteristic clinical features of PD including bradykinesia, rigidity and tremor. These applications are centrally reviewed in the SII. Special reimbursement for PD medications is granted if predefined criteria for PD diagnosis are fulfilled and diagnoses must be confirmed by a neurologist. Diagnosis of PD was based on United Kingdom Parkinson's Disease Society Brain Bank's criteria [18].

An age (+/- 1 year), sex and region-matched comparison cohort was identified from the SII database covering all residents. The index date was the date of PD diagnosis for the matched referent. The comparison people were not allowed to have purchases of PD medication (Anatomical Therapeutic Chemical classification ATC code N04) or the reimbursement code ever before the index date or 12 months after and during the diagnosis month of the referent people with PD. They also had to remain alive and community-dwelling during the month of index date. The diagnosis-based exclusion criteria of comparison people was otherwise similar to that of the PD cohort, but dementia due to PD (ICD-10 F02.3) was added to list of exclusion criteria, leaving altogether 148, 009 comparison people. Dementia due to PD was added to ensure exclusion of people with PD from the comparison cohort.

Incidence of antidepressant use was investigated from 10 years before to 15 years after the PD diagnosis. Data on antidepressant purchases during 1995-2016 were gathered from the Prescription Register, which contains data on reimbursed medication purchases. Antidepressants were defined as ATC class N06A (Supplementary Table 2), and further categorized as selective serotonin reuptake inhibitors (SSRIs), tricyclic antidepressants (TCAs), serotonin-norepinephrine reuptake inhibitors (SNRIs), mirtazapine, and other antidepressants. Incident users were identified with a one-year washoutperiod, starting 11 years before PD diagnosis. For those diagnosed before or during the year 2005, year 1995 was used as a one-year washout-period. People who purchased antidepressants during the washout period, those who were hospitalised for $>50 \%$ of the washout or 
hospitalised for the last 90 days of washout were excluded (Supplementary Figure 1). Only the first initiation of antidepressant use after washout period was included in the analysis. Hospitalisation data were obtained from the Care Register for Health Care.

Data on comorbidities since 1972 until the index date were obtained from the Special Reimbursement register: asthma or chronic obstructive pulmonary disease (code 203), cardiovascular diseases including chronic heart failure (201) hypertension (205), coronary artery disease $(206,213,280)$ and rheumatoid arthritis and connective tissue diseases (code 202). Diabetes was defined as special reimbursement code 103 or purchase of antidiabetics (ATC A10, excluding guar gum A10BX01). History of depression (discharge diagnosis or diagnosis in outpatient visit in specialized health care) was identified from the Care Register for Health Care using ICD-82960, 3004, 3011; ICD-9: 2961, 2968, 3011, 3004; ICD-10 F32F34, F38-F39.

\section{Statistical analyses}

The incidence rate (IR) of antidepressant initiations per 100 person-years was calculated for every six-month period starting from 10 years before and ending 15 years after the index date. The IRs were compared with Poisson regression between persons with PD and their comparison persons and reported as incidence rate ratios (IRR) with 95\% confidence intervals (CI). As the Prescription register does not contain data on drugs used in hospitals or public nursing homes, people with 120 or more hospital days within a six-month period were excluded from that period.

The follow-up ended on initiation of antidepressant use, death, end of study follow-up (15 years after PD diagnosis) or end of data linkage (31.12.2016), whichever occurred first. In addition, comparison people who received PD diagnosis during the follow-up $(n=79)$ were censored on the date of diagnosis. Altogether 75 of these comparison people were diagnosed with PD during 1996-2015 so they were also included in the PD group. The results were similar after excluding these 75 people from the comparison group (data available from the corresponding author by request). The main analyses included all people, regardless of year of PD diagnoses but we performed sensitivity analysis on initiation of any antidepressant for those who had at least 10 years of purchase data prior to PD diagnosis (PD diagnosed in 2006-2015).

Characteristics of antidepressant initiators and noninitiators, and initiators with and without PD were compared with the chi-squared test for categorical variables and with $t$-test for the continuous variables. The analyses were performed using Stata MP 14.0.

\section{Results}

The final study sample included 20,546 people with and 140,291 people without PD. During the study period, antidepressant initiation was more common among people with PD $(n=9091,44.3 \%)$ compared to people without PD $(n=35,083,25.0 \%)$. Women were more likely to initiate antidepressants in both groups. Association between baseline age and antidepressant initiation was not clinically significant, but people with PD were 1 year younger when they initiated antidepressants compared with persons without PD (Table 1). Depression was the only comorbidity that was more common among initiators with PD in comparison to initiators without PD (10.2\% vs. $7.6 \%)$. In persons with and without $\mathrm{PD}$, the most frequently initiated antidepressant group was SSRIs. SSRIs and mirtazapine together covered $81.0 \%$ of all initiations among users with PD and 78.7\% among users without PD.

Among people with $\mathrm{PD}$, the incidence was comparable to that of the comparison cohort until 7 years before PD diagnosis, when the difference began to emerge. The difference remained until the end of the follow-up, although the confidence intervals of the PD cohort widened, and overlapped with the comparison group at timepoint 13 years after PD diagnosis (Fig. 1a, Supplementary Table 3). Among people without PD the incidence of antidepressant initiation was approximately two per 100 person-years throughout the study period. The largest difference was observed 6 months before PD diagnosis (IRR 5.28 , 95\% confidence interval 4.80-5.55), with 9.02 initiations per 100 person-years among PD persons compared to 1.68 per 100 person-years among people without PD. Similar incidence peak and increase 7 years before PD diagnosis was observed in sensitivity analyses restricted to those with at least 10 year of purchase data prior to PD diagnosis (Supplementary Figure 2).

The incidence of SSRIs initiations followed similar trends with any antidepressant initiations, with the highest incidence observed 6 months before PD diagnosis (Fig. 1b). Similar incidence peaks, but at lower level were observed for mirtazapine and TCAs (Fig. 1c-d). There was a continuous upward trend in mirtazapine initiations in people with and without PD. The initiation of SNRIs and other antidepressants were less common, and the differences between people with and without PD were small (Fig. 1e-f).

\section{Discussion}

Our study shows that the incidence of antidepressant use in people with PD began to increase already 7 years before the diagnosis, with peak occurring around 6 months before diagnosis. The incidence remained above the comparison group for over 10 years after PD diagnosis. These findings extend the previous evidence on 
Table 1 Comparison of incident antidepressant initiators and noninitiators with and without PD. Comorbidities and university hospital district represent data on index date (date of Parkinson's disease diagnosis)

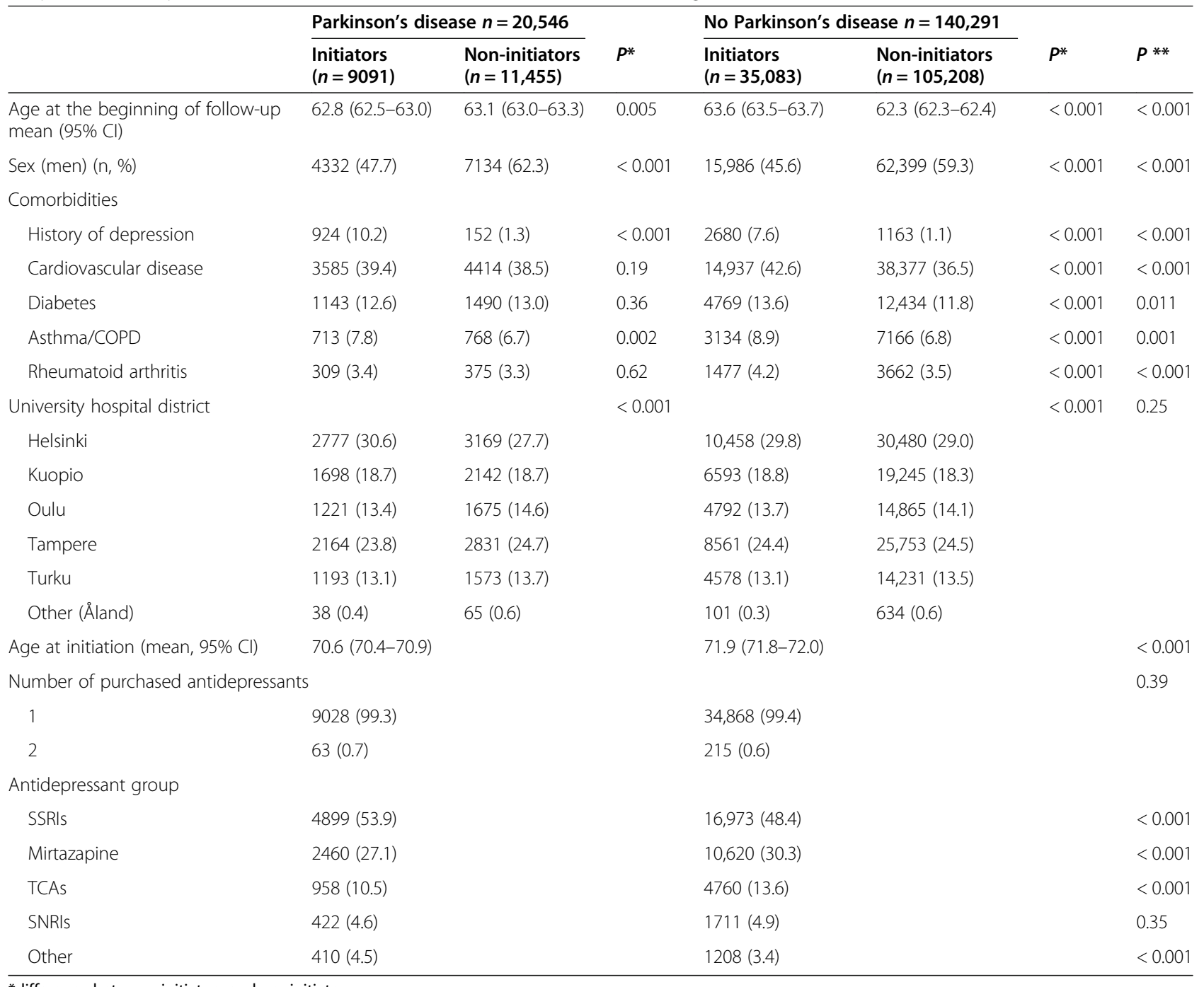

*difference between initiators and noninitiators

** difference between initiators with and without PD

higher prevalence of depression and depressive symptoms in people with PD [3-6], and studies showing higher prevalence and incidence of antidepressants in antiparkinson drug users $[13,14]$.

The added value of our study to previous studies on incidence and prevalence of antidepressant use in antiparkinson drug users $[13,14]$ or people with in- or outpatient hospital care with PD diagnosis [15] is providing more detailed information on temporal changes in antidepressant initiation in relation to PD diagnosis. A cross-sectional study compared the prevalence of antidepressant use between users and nonusers of dopaminergic antiparkinson drugs in one three-month time-window [13]. Thus, it did account for duration of antiparkinson drug use. The two studies which assessed incidence of antidepressant use after initiation of any dopaminergic antiparkinson drug [14] or hospital care (in- or outpatient) with PD as main diagnosis [15] reported the incidence for three different time windows (0-6 months, 6-12 months and over 1 year). Thus, it was not previously known when the difference emerges and how long it remains. In the two previous studies on the incidence of antidepressant use, the initiation rates peaked during the first 6 months after antiparkinson drug initiation or hospital admission with PD as main diagnosis $[14,15]$. However, these studies began the follow-up from the initiation of antiparkinson drug or hospitalisation and due to these differences in study design they are not comparable with our study.

In addition, the use of dopaminergic antiparkinson drugs $[13,14]$ or secondary healthcare admission with $\mathrm{PD}$ as a main diagnosis [15] as a proxy for PD in these 


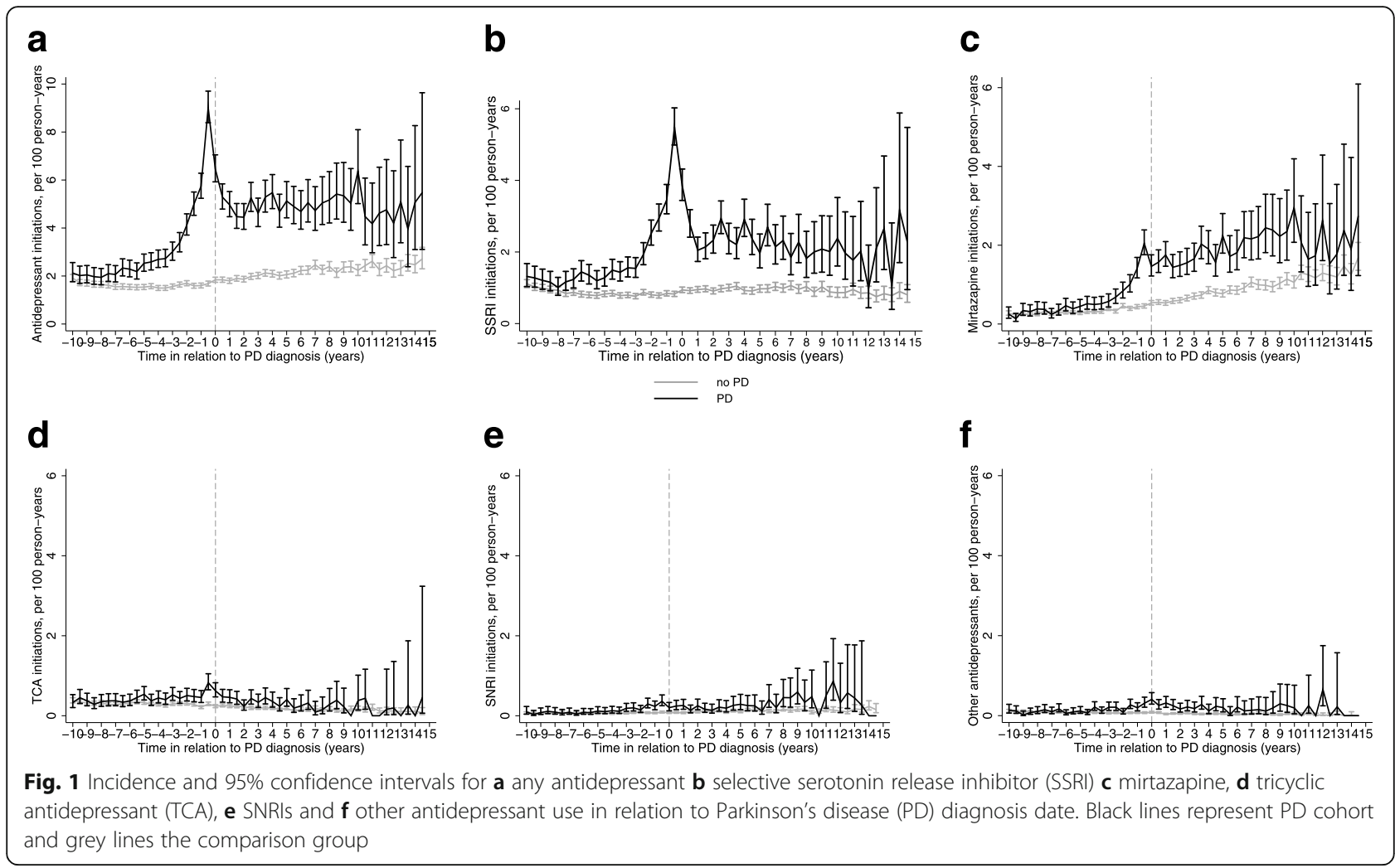

previous studies may complicate the comparison of our results to these previous studies. Diagnosis of PD is challenging, as indicated by the relatively large proportion of false diagnoses $[16,17]$. Treatment trial on antiparkinson drugs is often a part of the diagnostic process, and these drugs can be used for other reasons, ranging from restless legs to rare cases of stopping milk production after labour. Therefore, previous studies excluded women aged 40 years or younger using bromocriptine and cabergoline [14] and restricted the studies to users of dopaminergic drugs $[13,14]$.

As our definition of PD was based on clinically confirmed diagnosis instead of antiparkinson drug use, it includes also those people who received the diagnosis regardless on whether or not they initiated the antiparkinson drugs on that specific date. People who likely initiated antiparkinson drugs for other reasons were not included. The proportion of people excluded due to possible misdiagnoses in our cohort is within the range of estimated proportion of false diagnoses $[16,17]$.

The increase in incidence rate already 7 years before PD diagnosis in our study is novel observation supported by the extending literature on non-motor symptoms including depression, anxiety and sleep disorders, which can occur years before PD diagnosis, and even before the motor symptoms [3-6]. According to a systematic review, history of depression is more common in people with PD [3], and a study based on the Health
Improvement Network (THIN) database showed that depression, defined as Read code or antidepressant prescription was more common in people with PD already 5 years before diagnosis [4]. The higher incidence after PD diagnosis in our study may be explained by the high prevalence $(40-50 \%)$ of depression in people with PD as depression is the most frequently reported neuropsychiatric disturbance in PD. [7] However, it should be noted that the CIs at the end of follow-up were wide because the number of eligible initiators had become small and in reality, the difference may remain longer.

SSRIs were the most frequently initiated antidepressants. This is expected, as according to a meta-analysis of different antidepressants groups, only SSRIs had significant effect on depression in PD [19]. In addition to mood disorders like depression [7], also anxiety and sleep disorders [3] as well peripheral neuropathic pain [20] are more common in people with $\mathrm{PD}$, so these conditions may also explain the results. SNRIs and TCAs can be used to treat neuropathic pain, but their use was low in our study population. Sleep disorders may explain the increased initiation of mirtazapine during the follow-up.

We acknowledge that one limitation of our study is the lack of data on indication for antidepressant initiation. Thus, assessing the indications and acknowledging possibly changing indications during the disease course would be important in future studies. In our study, the prevalence of hospital-treated depression was $10.6 \%$ in 
antidepressant initiators with PD. Although this covers only the most severe cases of depression, it is unlikely that depression or depressive symptoms entirely explain the increase in incidence.

In conclusion, the initiation of antidepressants among people with PD became more common already 7 years before the PD diagnosis and continued to remain above comparison group more than 10 years after diagnosis. Persons with PD have neuropsychiatric symptoms such as depression that may require antidepressant treatment years before and after diagnosis. The symptoms needing antidepressant treatment may be clinical signs of possible PD and they should be considered as a need to assess clinical status in person diagnosed with PD.

\section{Abbreviations}

ATC: Anatomical therapeutic chemical classification; FINPARK: Finnish Study on Parkinson's disease; IR: Incidence rate, IRR incidence rate ratio; PD: Parkinson's disease; SII: Social Insurance Institution of Finland; SNRI: Serotonin-norepinephrine reuptake inhibitor; SSRI: Serotonin reuptake inhibitor; TCA: Tricyclic antidepressant

\section{Supplementary Information}

The online version contains supplementary material available at https://doi. org/10.1186/s12877-021-02145-6

Additional file 1: Supplementary Table 1. Exclusion diagnoses for the PD cohort. Supplementary Table 2. Antidepressants and ATC-codes. Supplementary Table $\mathbf{3}$. Incidence rates of antidepressant initiation in people with and without Parkinson's disease in different time periods. Supplementary Figure 1. Formation of study population. Supplementary Figure 2. Incidence of any antidepressant use in those with at least 10 years of purchase data before the index date (index date 1.1.200631.12.2015).

\section{Acknowledgements}

Not applicable.

\section{Authors' contributions}

EH: execution of research project, execution and review of statistical analysis, writing of the first draft, review and critique of manuscript. MT: statistical analysis review and critique, review and critique of manuscript. HT: statistical analysis review and critique, review and critique of manuscript. $\mathrm{SH}$ : research project conception and organization, review and critique of manuscript. AMT: research project conception and organization, statistical analysis design, execution, review and critique, review and critique of manuscript. The author(s) read and approved the final manuscript.

\section{Funding}

None.

\section{Availability of data and materials}

The data that support the findings of this study are available from the corresponding author but restrictions apply to the availability of these data, and so they are not publicly available. Data are however available from the authors upon reasonable request and with permission of the register maintainers.

\section{Declarations}

\section{Ethics approval and consent to participate}

Register maintainers have approved the FINPARK study plan. Data were pseudonymized before submission to the research team and study participants were not contacted. Therefore, according to Finnish legislation (including Personal Data Act 23/1999, Act on the Openness of Government
Activities 621/1999 and Act on the Secondary Use of Health and Social Data 552/2019 (and previous Act on the National Healthcare registers, not official English translation as this is not available 556/1989) the study has been granted an exemption from requiring ethics approval or informed consent.

\section{Consent for publication}

Not applicable.

\section{Competing interests}

$\mathrm{SH}$ reports lecture fees from Astellas Pharma. HT acknowledges personal fees from Janssen Cilag and research grants from Janssen Cilag and Eli Lilly, outside of the submitted work. EH, MT, AMT have nothing to declare.

\section{Author details}

${ }^{1}$ School of Pharmacy, University of Eastern Finland, P.O. Box 1627, 70211 Kuopio, Finland. ${ }^{2}$ Kuopio Research Centre of Geriatric Care, University of Eastern Finland, P.O. Box 1627, 70211 Kuopio, Finland. ${ }^{3}$ Department of Clinical Neuroscience, Karolinska Institutet, Stockholm, Sweden. ${ }^{4}$ Department of Forensic Psychiatry, Niuvanniemi Hospital, University of Eastern Finland, Kuopio, Finland.

Received: 17 December 2020 Accepted: 10 March 2021

Published online: 23 March 2021

References

1. Tysnes O-B, Storstein A. Epidemiology of Parkinson's disease. J Neural Transm (Vienna). 2017;124(8):901-5. https://doi.org/10.1007/s00702-01 7-1686-y.

2. Schapira $A H V$, Chaudhuri KR, Jenner $P$. Non-motor features of Parkinson disease. Nat Rev Neurosci. 2017;18(8):509. https://doi.org/1 0.1038/nrn.2017.91.

3. Ishihara L, Brayne C. A systematic review of depression and mental illness preceding Parkinson's disease. Acta Neurol Scand. 2006:113(4):211-20. https://doi.org/10.1111/j.1600-0404.2006.00579.x.

4. Schrag A, Horsfall L, Walters K, Noyce A, Petersen I. Prediagnostic presentations of Parkinson's disease in primary care: a case-control study. Lancet Neurol. 2015;14(1):57-64. https://doi.org/10.1016/\$14 74-4422(14)70287-X

5. Noyce AJ, Bestwick JP, Silveira-Moriyama L, Hawkes $C H$, Giovannoni G, Lees AJ, Schrag A. Meta-analysis of early nonmotor features and risk factors for Parkinson disease. Ann Neurol. 2012;72(6):893-901. https://doi.org/10.1002/a na.23687.

6. Stern MB, Siderowf A. Parkinson's at risk syndrome: can Parkinson's disease be predicted? Mov Disord. 2010;25(Suppl 1):S89-93. https://doi.org/10.1002/ mds.22719.

7. Reijnders JSAM, Ehrt U, Weber WEJ, Aarsland D, Leentjens AFG. A systematic review of prevalence studies of depression in Parkinson's disease. Mov Disord. 2008;23(2):183-9; quiz 313. https://doi.org/10.1002/mds.21803.

8. Leentjens AFG, Dujardin K, Marsh L, Martinez-Martin P, Richard IH, Starkstein SE, Weintraub D, Sampaio C, Poewe W, Rascol O, Stebbins GT, Goetz CG. Anxiety rating scales in Parkinson's disease: critique and recommendations. Mov Disord. 2008;23(14):2015-25. https://doi.org/10.1002/mds.22233.

9. Kirsch-Darrow L, Fernandez HH, Fernandez HF, Marsiske M, Okun MS, Bowers D. Dissociating apathy and depression in Parkinson disease. Neurology. 2006;67(1):33-8. https://doi.org/10.1212/01.wnl.0000230572. 07791.22.

10. Marsh L. Neuropsychiatric aspects of Parkinson's disease. Psychosomatics. 2000;41(1):15-23. https://doi.org/10.1016/S0033-3182(00)71169-8.

11. Hantz P, Caradoc-Davies G, Caradoc-Davies T, Weatherall M, Dixon G. Depression in Parkinson's disease. Am J Psychiatry. 1994;151(7):1010-4

12. Cummings JL. Depression and Parkinson's disease: a review. Am J Psychiatry. 1992;149(4):443-54. https://doi.org/10.1176/ajp.149.4.443.

13. Haasum Y, Fastbom J, Johnell K. Use of antidepressants in Parkinson's disease: A Swedish register-based study of over 1.5 million older people. Parkinsonism Relat Disord. 2016;27:85-8.

14. Brandt-Christensen M, Kvist K, Nilsson FM, Andersen PK, Kessing LV. Treatment with antiparkinson and antidepressant drugs: a register-based, pharmaco-epidemiological study. Mov Disord. 2007;22(14):2037-42. https:// doi.org/10.1002/mds.21472. 
15. Brandt-Christensen M, Garcia Lopez A, Mørkeberg Nilsson F, Kragh Andersen P, Vedel KL. Parkinson's disease and antidepressant drug treatment: a caseregister study. Parkinsonism Relat Disord. 2007 Oct;13(7):406-10. https://doi. org/10.1016/j.parkreldis.2007.01.006.

16. Joutsa J, Gardberg M, Roytta M, Kaasinen V. Diagnostic accuracy of parkinsonism syndromes by general neurologists. Parkinsonism Relat Disord. 2014;20(8):840-4. https://doi.org/10.1016/j.parkreldis.2014.04.019.

17. Rizzo G, Copetti M, Arcuti S, Martino D, Fontana A, Logroscino G. Accuracy of clinical diagnosis of Parkinson disease: a systematic review and metaanalysis. Neurology. 2016;86(6):566-76. https://doi.org/10.1212/WNL. 0000000000002350.

18. Hughes AJ, Daniel SE, Kilford L, Lees AJ. Accuracy of clinical diagnosis of idiopathic Parkinson's disease: a clinico-pathological study of 100 cases. J Neurol Neurosurg Psychiatry. 1992;55(3):181-4. https://doi.org/10.1136/jnnp. 55.3.181.

19. Bomasang-Layno E, Fadlon I, Murray AN, Himelhoch S. Antidepressive treatments for Parkinson's disease: a systematic review and meta-analysis. Parkinsonism Relat Disord. 2015;21(8):833-42; discussion 833. https://doi. org/10.1016/.jparkreldis.2015.04.018.

20. Blanchet PJ, Brefel-Courbon C. Chronic pain and pain processing in Parkinson's disease. Prog Neuropsychopharmacol Biol Psychiatry. 2018;87(Pt B):200-6.

\section{Publisher's Note}

Springer Nature remains neutral with regard to jurisdictional claims in published maps and institutional affiliations.

Ready to submit your research? Choose BMC and benefit from:

- fast, convenient online submission

- thorough peer review by experienced researchers in your field

- rapid publication on acceptance

- support for research data, including large and complex data types

- gold Open Access which fosters wider collaboration and increased citations

- maximum visibility for your research: over $100 \mathrm{M}$ website views per year

At $\mathrm{BMC}$, research is always in progress.

Learn more biomedcentral.com/submissions 